\title{
Study on the Effect of Bell Mouth Structure to Its Outlet Flow Field Evenness
}

\author{
Zhi-Peng LI*, Guo-Wang ZHANG and Chao LU
}

AECC Shenyang Engine Research Institute, No. 1, Wanlian Road, Shenhe District, Shenyang, China

*lizhipengpdf@sina.com

Keywords: Bell mouth junction, CFD, AIP, Total pressure, Remodelling schemes.

\begin{abstract}
In this article a bell mouth junction assembled in front of the test article in practice was simulated by applying ANSYS CFX, the data of relative measurement points was picked up from the simulation result and compared with test data to validate the simulation reliability. Then, aiming to dwindle in the formal diameter of a standard designed bell mouth junction to satisfy assembling inside a small-diameter pipe in front of the test article, three remodeling schemes are introduced, and the validated simulation method is applied for the three. By comparing the simulation results, the effect of bell mouth inner wall profile to its outlet flow field evenness is obtained, furthermore, the location of aerodynamic interface plane (AIP) along the axis direction of junction is confirmed, and the optimized one of the three is elected.
\end{abstract}

\section{Introduction}

When processing a test for the compressor of an aeroengine, a bell mouth junction is generally assembled inside a straight pipe right in front of the test article inlet [1-4]. The facility is used to commute the intake air in a certain level and by the impact tubes assembled on AIP, total pressure of inlet is able to be obtained. Occasionally the inner wall profile of the bell mouth cannot be designed satisfying the relative standard, since its maximal formal diameter is barely too large to be embedded in when limited by the size of the straight pipe, for this reason the bell mouth has to be remodeled properly to satisfy this condition. Meanwhile, some technology methods should be taken to validate the schemes to reduce time and cost. In this article a bell mouth junction in practice in a high-pressure compressor test is simulated, the simulation result is taken to compare with test data with the parameters of relative measure points. It shows that there is enough accuracy for the simulation, which means the simulation method is reliable. Then, totally three types of remodeling schemes are imposed on designing a bell mouth with certain nominal diameter, which does not totally answer for the relative standard. Simulations are taken to obtain the flow field inside the junction, and compare the simulating results to select an optimized one.

\section{Standard Bell Mouth Junction Design Method and Remodelling Schemes}

The structure of the bell mouth junction can be seen in Fig.1.

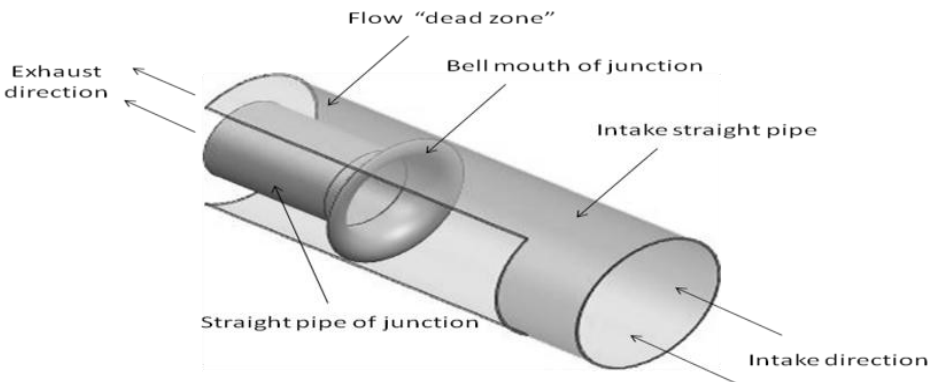

Figure 1. Structure of the bell mouth junction 
In the relative standard of compressor component test, the inner wall profile of a bell mouth and its construction formulation are given definitely, as is shown in Fig.2 and equation 1.

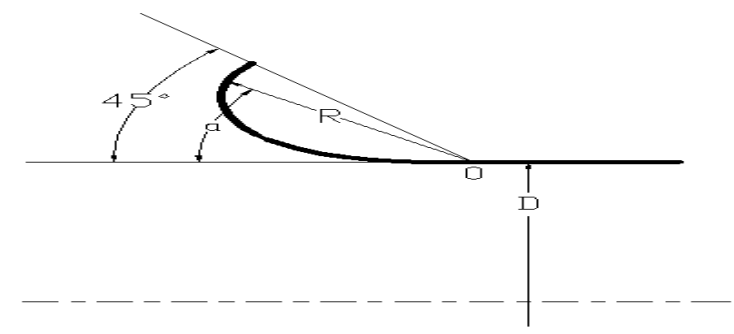

Figure 2. Sketch of the bell mouth inner wall profile

$$
R=a \cos 2 \alpha
$$

Where $0.6 D<a<0.8 D$ and $\alpha=0 \sim 45^{\circ}$

Some bell mouth inner wall profiles are shaped as one part of an ellipse [6], this kind of profile has no more description here since it is not common used in China.

When given the nominal diameter of the junction, by equation 1 the bell mouth inner wall profile parameters can be obtained easily by a certain way. In this article a small MATLAB is programmed and applied to act this. The program is not given out here since it is much too simple.

Equation 1 can be transformed into the following format to be more easily access to parameterized remodeling:

Name $a=k D$, then equation 1 can be transformed into equation 2

$$
R=k D \cos 2 \alpha
$$

Where, $k \subset(0.6,0.8), \alpha=0 \sim 45^{\circ}$.

According to equation 2, there are to controlled variables $k$ and $\alpha$ to be used to adjust the parameters of junction inner wall profile. In this article reducing the values of the two variables to dwindle the maximal formal diameter of the bell mouth in a smaller one is applied, the two methods are named method of cutdown (scheme 1) and method of cutoff edge (scheme 2).

In an engineer practice, making $k<0.8$ is mainly to reduce the size of the junction, make sure the size is not too large to be easily assembled. However, it changes to be not essential since only maximal formal diameter of the bell mouth is required to be smaller than that of the straight pipe. In fact, a larger $k$ may lead to a better effect of commuting effect since the shrinkage of the bell mouth inner wall profile becomes smoother. For this reason, in this article a third method of remodeling is introduced, namely using a value of $k$ larger than 0.8 meanwhile reduced the range of $\alpha$, which is called method of cutoff edge with enlarged outline (scheme 3 ).

The inner wall profiles of the three remodeling schemes are shown in Fig. 3, all of the three schemes are simulated respectively in this article and the calculating results are compared in the following paragraphs.

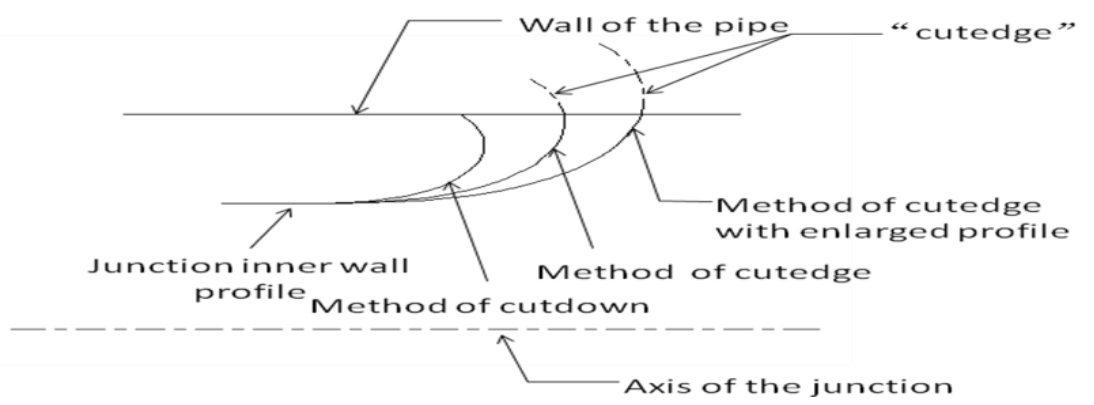

Figure 3. The inner wall profiles of the three remodeling schemes 


\section{Boundary Conditions Set Up and Simulation Reliability Validating}

As what is shown in Fig.1, in the downstream there is a flow "dead zone", during the simulation this region can be neglected. After that the research aim turns to the flow inside the straight pipe and junction, as is shown in Fig.4.

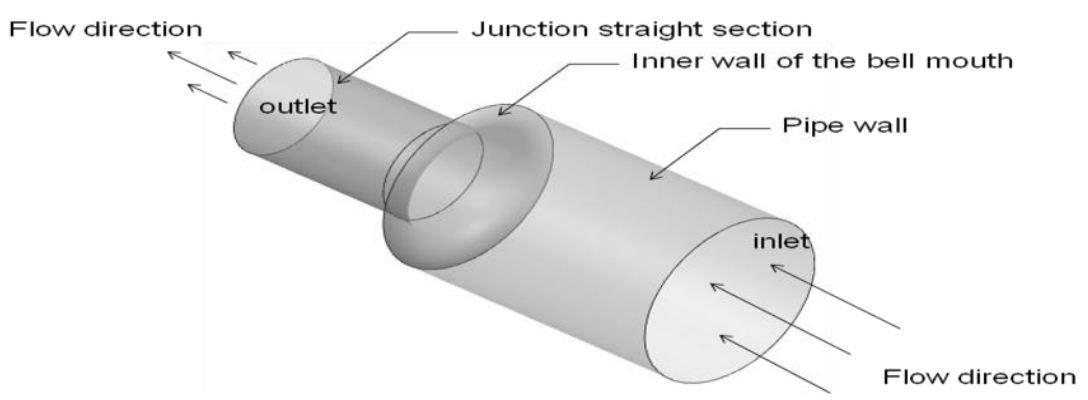

Figure 4. Sketch of the bell mouth junction model

First a bell mouth in practice in a test is meshed according to the structure of Fig.4, the meshing software is ANSYS CFX, and the type of mesh is structural meshes (hexahedron mesh). The meshing conditions of remodeling schemes will not be described in the following paragraphs since it is the most difficult for meshing the practical one, which can be seen in Fig.5.

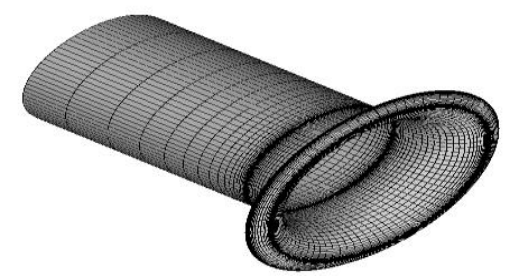

Figure 5. Meshing conditions (only meshes for bell mouth are shown)

As what is calculated in ICME, the number of mesh elements is about 270,000, while that of nods is 310,000 , they are nearly the same, the lowest quality of meshes is 0.2 , and only $8 \%$ of meshes have quality lower than 0.5 , which means the meshing condition satisfies the simulation's requirement.

With Fig.2, initial boundary conditions are set up as what are shown in Table 1:

Table 1 . Initial boundary condition set up

\begin{tabular}{|c|c|}
\hline Name of the boundary & Boundary condition \\
\hline Inlet & Inlet, total pressure \\
\hline outlet & Outlet, velocity $($ Ma number $)$ \\
\hline others & No slip wall, smooth wall \\
\hline
\end{tabular}

The boundary conditions in Table1 also are also accepted by the other cases, which will not be described in the following paragraphs.

Fig.6 gives the impact tubes of inlet assembled in practice in the compressor test. In this test there are two impact tubes to process total pressure measurement at inlet of the test article. The relative total pressure data are equally the same since the two impact tubes are assembled face to face, and that's why in this article only data of the test from one of the two is given, see Table2. What needs to be informed is that all the data is the ratio of what to point 1 . Table 2 also gives out the data from simulation results, treated in the same way, with deviation in the end.

From Table 2 it can be seen that deviations between test and simulation data are about $3 \%$ in all, so it can be confirmed that the simulation method is reliable. 
What needs to be illuminated is that the flow can be considered as adiabatic, so during the simulation no temperature change is taken into account.

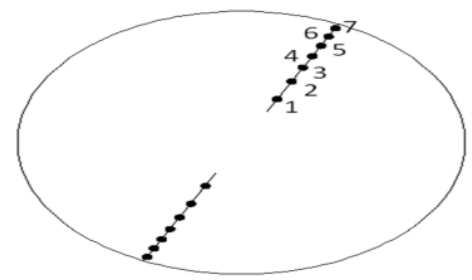

Figure 6. Impact tubes distribution at inlet

Table 2. Comparison between data of simulation and test

\begin{tabular}{|l|c|c|c|c|c|c|c|}
\hline Point number & $\mathbf{1}$ & $\mathbf{2}$ & $\mathbf{3}$ & $\mathbf{4}$ & $\mathbf{5}$ & $\mathbf{6}$ & $\mathbf{7}$ \\
\hline Test data & 1 & 0.999601 & 1.008603 & 0.998574 & 0.999864 & 0.997289 & 0.995975 \\
\hline Simulation data & 1.028933 & 1.028934 & 1.028936 & 1.028937 & 1.028939 & 1.028941 & 1.024902 \\
\hline Deviation (\%) & $2.893 \%$ & $2.935 \%$ & $2.016 \%$ & $3.041 \%$ & $2.908 \%$ & $3.174 \%$ & $2.904 \%$ \\
\hline
\end{tabular}

\section{Simulation Results and Analysis}

In the following sections the simulation results of the three remodeling schemes are given. To compare the influence to the flow inside the junction with by different outlet $M a$ numbers, $M a=0.2$ and $M a=0.6$ are given to each scheme.

First scheme1 is described as an example to compare the flow fields with different outlet Ma numbers.

\section{The Influence of Different Ma Numbers to the Flow Field Distribution}

The static pressure distribution inside the junction is required to be considered when analyzing the effect of remodeling, in this way first the static pressure distribution contours on the longitudinal section of the junction are given out in a) and b) of Fig.7. Considering that different outlet Ma numbers can lead to distinct static pressure, which is not able to get well defined arrangements of static pressure variation, here no uniform legend is used for the two figures in Fig.6, namely each figure is imposed its own range of pressure.

From Fig.7 it can be seen that the outlet $M a$ number does not influent the varying regular of static pressure inside the junction, but only makes slight difference on the beginning and final axis locations where static pressure changes. Picking up the value of static pressure gradient along the axis from results with different outlet Ma numbers the varying curves in Fig. 8 can be obtained:

Fig. 8 gives out changing curve of static pressure gradient along axis. In practice, the location of AIP is frequently selected downstream of junction throat (namely the connection plane between the bell mouth and the straight section of the junction). On AIP the static pressure should keep constant, namely the pressure gradient at the point of intersection by the axis and AIP should no more change after a certain place. In Fig.7 it shows that bigger Ma number makes the proper AIP move along the axis to the outlet of junction, and a slight wave of pressure gradient, but not so much to influent the accuracy of measurement.

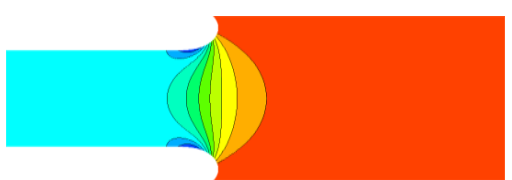

a) $\mathrm{Ma}=0.2$

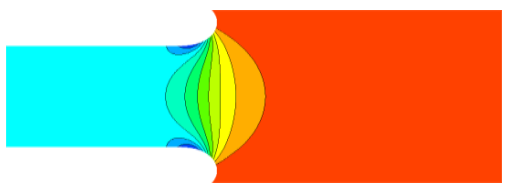

b) $\mathrm{Ma}=0.6$

Figure 7. Static pressure distribution contours on the longitudinal section (different outlet Ma Number) 


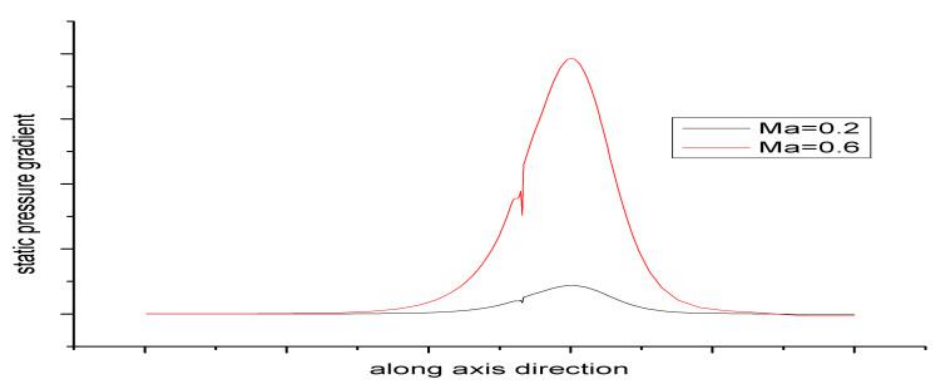

Figure 8. Changing curve of static pressure gradient along axis

\section{Comparison among Different Remodelling-Scheme Simulating Results and Analysis}

Here the way of analyzing is the same as what is applied above, but all the legends are unified since same outlet Ma numbers can make only slight differences of pressure inside each kind of junctions.

Fig.9 gives out the static pressure distribution contours on longitudinal sections with different schemes, while $M a=0.2$.

From Fig.9 it can be seen that no distinct influence to pressure distribution on longitudinal section is raised by different remodeling schemes. Fig.9 gives out changing curves of pressure gradients along axis with different schemes, the initial plane is throat of the junction.

More information about static pressure change is given in Fig.10. In a distance downstream of throat, the three curves of static pressure changing coincide with each other, and fall with a small speed together. However, they separate then, the curve of scheme1 flatten out a little faster, and the ones of scheme 2 and scheme 3 go on together, flatten out after a sudden fall.

To explain this, the contours in Fig.9 are examined deeper, it can be seen that there is a lowpressure zone shaped like a blade near the wall of throat, the area of which of schemel is large than those of the others, and the value of static pressure is more distinct than that of the main flow, which may be the reason.

The static pressure changing curves at downstream of throat for scheme 2 and scheme 3 are together shows that the value of $k$ acts nothing to the remodeling, the two schemes are equal to each other in substance.

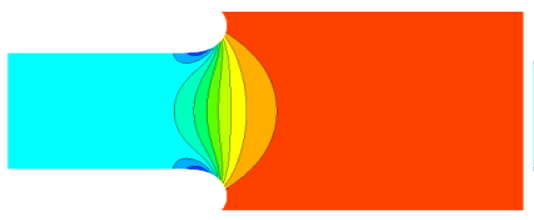

a) Scheme 1

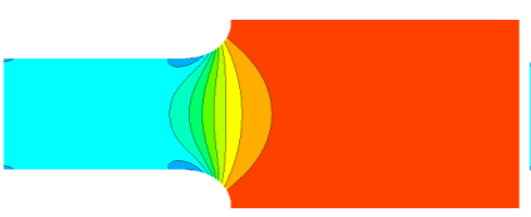

b) Scheme 2

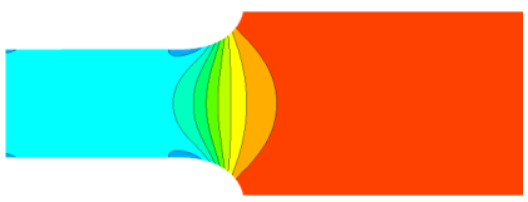

c) Scheme 3

Figure 9. Static pressure distribution contours on longitudinal sections with different schemes, $\mathrm{Ma}=0.2$

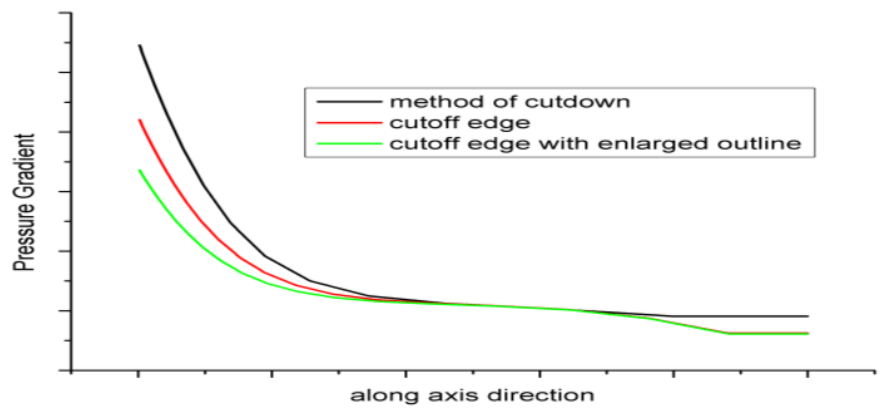

Figure 10. Changing curves of pressure gradient alond axis direction $(\mathrm{Ma}=0.2)$ 
Fig.11 gives out the static pressure distribution contours on longitudinal sections with different schemes, while $M a=0.6$.

Fig.12 gives out changing curves of pressure gradients along axis with different schemes, the initial plane is throat of the junction.

No more analysis is given for Fig.11 and Fig.12 since they are the same to Fig.9 and Fig.10 respectively in tendency, just with different values.

Furthermore, by summarizing data of Fig.10 and Fig.12, it can be found that for scheme1, the distance between AIP and throat should be 1.2 times or larger of junction nominal diameter; while for scheme 2 and 3 the distance should not smaller than 1.32 times of junction nominal diameter, or the flux measured and calculated on AIP will be smaller than the practical outlet of the junction. Since in a test it is hoped to shorten the intake distance by smaller length of junction straight section, scheme1, namely method of cutdown is the optimized way for remodeling.

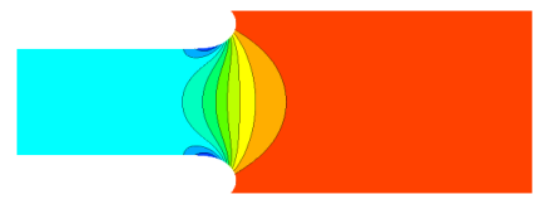

a) Scheme 1

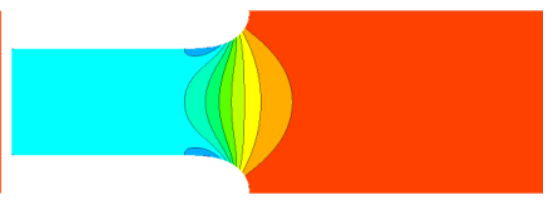

b) Scheme 2

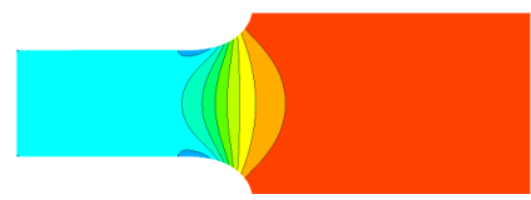

c) Scheme 3

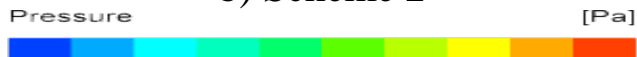

Figure 11. Static pressure distribution contours on longitudinal sections with different schemes, $\mathrm{Ma}=0.6$

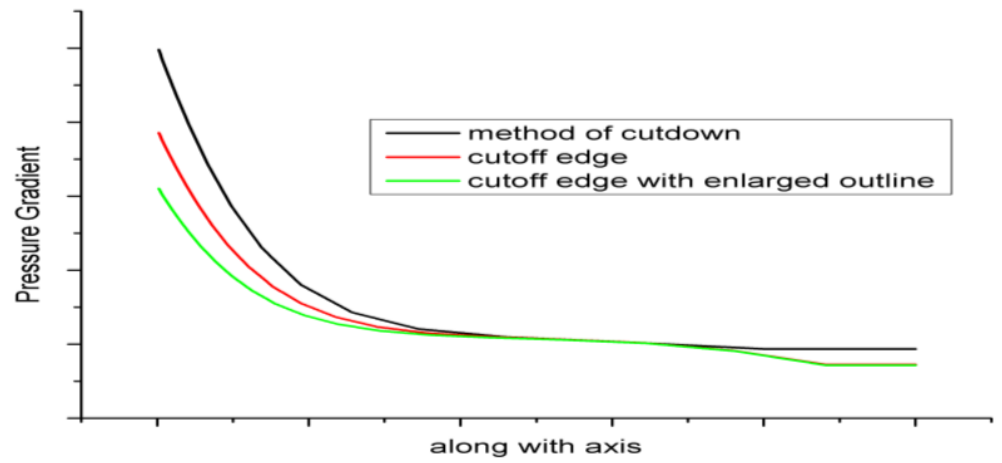

Figure 12. Changing curves of pressure gradient alond axis direction $(\mathrm{Ma}=0.6)$

\section{Conclusions and Prospects}

In this method the simulation method is first validated by comparing with data of simulating a bell mouth junction in practice in a test and from the test. The deviations between the two are just around 3\%, which shows the reliability of the simulation method. Secondly, three remodeling schemes for a standard designed bell mouth junction are given in this article to satisfy an intake straight pipe with smaller diameter, which are named method of cutdown, method of cutoff edge and method of cutoff edge with enlarged outline. A MATLAB program is applied to obtain the parameters of inner wall profiles of different scheme. Then, all of the schemes are simulated by the simulation method being validated, combined with one scheme with different outlet Ma numbers, conclusions of the simulations are given below:

(a)Outlet Ma number does not influent the changing characteristics of static pressure, but bigger Ma number leads to a slight wave of static pressure gradient along the axis of the junction, although not too much to make a accuracy loss of measurement;

(b)Compare to the other two schemes, by the method of cutdown, the distance between the selected AIP and throat plan is shorter, which means that applying this method the speed of becoming even at outlet of the junction is faster, leading to a shorter straight section of the junction, for this reason the method of cutdown is optimized; 
(c)Having to apply the method of cutoff edge or cutoff edge with enlarged outline for some certain reasons, the changing curves of static pressure gradient along the axis are together in downstream of the throat for both, which means that no difference between the two schemes in substance. When using method of cutoff for remodeling, the value of $k$ plays no role in changing the static pressure varying characteristics.

In this article only three schemes of remodeling are introduced. There must be some other schemes which are not included here, so the method of cutdown cannot be defined as the best one. In the later study, other schemes may be introduced to get a better scheme.

CFD technology has been applied in this article to process flow simulation for the remodeling junction, this validating method has been widely used in engineering practice to reduce design time and cost, furthermore get a complete understanding of the flow field to make a deeper analysis.

What needs to be pointed out is that although simulation is able to get a result with certain level of accuracy, rig test is essential to the design and data from test and simulation are required to be compared and validated for each other, even apply test data to adjust and modify the simulation to get a true output. This design process is also a general rule of positive design, which deserves to be referred and extended.

\section{References}

1. LIU Yi-cao, YANG Hua-dong, FAN Xiao-liang. Design of Transonic Single Stage Axial Compressor Test Rig. Machinery Design \& Manufacture. Shenyang. 2014;

2. Li Jiarui. Experimental and Numerical Simulation Study on a 1.5 Stage Compressor. Harbin Engineering University, Harbin, 2010;

3. Liu Bin. The Aerodynamic and Structural Design of Subsonic Repeating Stages Compressor Testing Rig, Dalian Maritime University, Dalian, 2015;

4. Cao Lei. Investigation into the Influence of Inlet Fogging on the Flow Field in Inlet System and Compressor, Harbin Engineering University, Harbin, 2011;

5. Zhao Quanchun, Duan Lianfeng, Shen Peiying, compressor aerodynamic performance test, Standard of aviation of People's Republic of China, HB7115-94, Shenyang, 1994;

6. D. K. Beale, T. L Hand, C. L Sebourn, Development of a Bellmouth Airflow Measurement Technique for Turbine Engine Ground Test Facilities, AIAA, Salt Lake City, 2001. 